\title{
LES CONDUITES ALLÉGEANTES ET LA REPRÉSENTATION DE LEUR VALORISATION SOCIALE SELON LE STATUT PROFESSIONNEL : UNE ÉTUDE RÉALISÉE DANS LA PROVINCE DE BUENOS AIRES
}

\author{
GANGLOFF Bernard ${ }^{11}$ \\ Université de Rouen (France) et Université Fédérale de Paraïba (Brésil) \\ MAYORAL Luisa ${ }^{22}$ \\ Université du Centre de la Province de Buenos Aires \\ ROMERO Maria del Carmen ${ }^{33}$ \\ Université du Centre de la Province de Buenos Aires
}

\begin{abstract}
La valorisation sociale d'une conduite et la dévalorisation de la conduite opposée constituent les caractéristiques essentielles d'une norme sociale. Il a été constaté, lors d'études récemment réalisées dans des entreprises de la province de Buenos Aires, que les cadres ayant du personnel à charge valorisaient les subordonnés allégeants, ce qui permet de penser à l'existence d'une norme d'allégeance, et que les subordonnés, ouvriers comme cadres (mais cadres sans charge de personnel), étaient conscients de cette norme.

Nous souhaitons ici savoir : 1) si les subordonnés ont une conscience de cette norme de manière globale ou s'ils effectuent des différenciations entre ses diverses concrétisations 2) si, spontanément, ils suivent cette norme en ses diverses concrétisations ou de manière sélective, et 3) si ces attitudes globalisantes ou différenciatrices des représentations et des conduites varient selon le statut (ouvrier ou cadre) du subordonné.

Nous avons, pour répondre à ces questions, utilisé le paradigme de l'autoprésentation surnormative versus contrenormative. C'est-à-dire que nous avons présenté à 180 salariés de la province de Buenos Aires (90 ouvriers et 90 cadres sans personnel à charge) une liste de conduites en leur demandant d'indiquer celles que, spontanément, ils étaient tentés d'adopter, celles qu'ils adopteraient s'ils souhaitaient donner une bonne image d'eux-mêmes, et celles qu'ils adopteraient pour en donner une mauvaise image.

Les résultats obtenus confirment nos hypothèses de sélectivité des réponses et d'effet de la variable statut.
\end{abstract}

The social valorization of a behavior and the depreciation of the opposite behavior constitute the essential features of a social norm. Recent studies realized in enterprises in the Buenos Aires region have proven that the allegiant subordinates are valorized by the load of staff managers. These findings permit us to believe that allegiance can exist under the form of a norm. Furthermore the subordinates, workers and managers also (but managers without load of staff), were conscious of this norm.

With this study we are trying to respond at the following questions: 1) The subordinates have a conscience of this norm in a global manner or they do some differentiations

\footnotetext{
${ }^{1}$ Adresa de contact: Laboratoire PRIS, Département de Psychologie, Université de Rouen (France) et Département de Postgraduation en Gestion à l'Université Fédérale de Paraïba (Brésil). Courrier électronique : bernard.gangloff@univ-rouen.fr

2 Université du Centre de la Province de Buenos Aires, Tandil (Argentine). Courrier électronique : Imayoral@eco.unicen.edu.ar

3 Université du Centre de la Province de Buenos Aires, Tandil (Argentine). Courrier électronique : romero@econ.unicen.edu.ar
} 
between its various materializations? 2) They follow this norm spontaneously in its various materializations or in a selective manner? 3) These globalized or differentiated attitudes of the representations and behaviors vary along the statute (worker or manager) of the subordinate?

To answer at these questions, we have used the paradigm of the over normative versus counter normative auto presentation. According to this paradigm we have presented to 180 employees of the province of Buenos Aires (90 workers and 90 managers without load of staff) a list of behaviors and have asked them to indicate those that, spontaneously, they were tempted to adopt, those that they would adopt if they wished to present a good image of themselves, and those that they would adopt to present a bad image.

The results obtained confirm our hypotheses of selectivity of the answers and effect of the statute.

Key-words: allégeance, recrutement, statut professionnel

\section{INTRODUCTION}

Toute situation de recrutement peut, pour un recruteur, se traduire par la question suivante: le candidat possède-t-il le bon profil ? Et l'on sait depuis longtemps que les aspects essentiels de ce profil concernent moins les compétences techniques que les compétences sociales, c'est-à-dire le savoirêtre du candidat. Ce savoir-être est notamment constitué de ce que l'on appelle la personnalité, et à l'heure actuelle il existe chez les psychologues un quasi-consensus pour considérer que cette personnalité peut être décrite grâce à 5 grandes dimensions, de 5 grands traits qui sont l'Energie, l'Amabilité, le Caractère consciencieux, la Stabilité émotionnelle, et l'Ouverture d'esprit ; c'est le modèle du Big Five (cf. Rolland, 1994, p 65 ; Caprara, Barbaranelli et Borgogni, 1997, p13). On observe alors (Gangloff, 2000) que le candidat idéal est généralement un candidat énergique (c'est-à-dire dynamique et dominant), aimable (faisant preuve de coopération et de cordialité), consciencieux (c'est-à-dire méticuleux et persévérant), émotionnellement stable (contrôlant ses émotions et ses impulsions), et à l'esprit ouvert (ouvert à la culture et à l'expérience).

Pour autant, il est récemment apparu qu'un autre critère de savoir-être, qu'un autre facteur, non personnologique mais normatif, a encore davantage d'importance que chacune de ces 5 dimensions personnologiques, a encore plus d'importance que la personnalité : il s'agit de l'adhésion à la norme d'allégeance. Cette adhésion se traduit par l'évitement de toute conduite de remise en cause de l'environnement social, et notamment de toute remise en cause de la hiérarchie des pouvoirs dans un système social donné (cf. pour une revue : Gangloff, 2002). L'individu adhérant à cette norme sera ainsi non seulement soumis à sa hiérarchie mais lui aura également prêté une sorte de serment de fidélité, évitant notamment toute conduite, en paroles ou en actes, pouvant lui porter quelque ombrage. Et il apparaît que les recruteurs préfèrent systématiquement un candidat non énergique, non aimable, non consciencieux, émotionnellement instable ou à l'esprit obtus mais faisant preuve d'allégeance, par rapport à un candidat possédant la personnalité idéale mais potentiellement rebelle (Gangloff et Huet, 2004). Enfin, il a aussi été observé que les candidats à un recrutement, ou plus globalement que les salariés sans position de commandement, sont conscients de cette préférence, sont conscients de la valorisation sociale de l'allégeance (cf. Gangloff, 2002).

$\mathrm{Si}$ la plupart des études précédemment citées sur la norme d'allégeance ont été conduites en France, des recherches complémentaires ont cependant également été menées en Argentine, avec des résultats similaires. II a notamment été observé que, dans les entreprises argentines, les cadres à responsabilités hiérarchiques préfèrent eux aussi travailler avec des subordonnés allégeants plutôt qu'avec des subordonnés potentiellement rebelles (Gangloff, Mayoral et Duringer, 2005 a). II fut aussi constaté que les subordonnés, ouvriers comme cadres (mais cadres moyens, qui sont donc, malgré leur statut de cadre, des subordonnés), ont une "clairvoyance normative" de l'allégeance (pour reprendre l'expression de Py et Somat, 1991), c'est-àdire qu'ils sont conscients de la valorisation de l'allégeance et que, spontanément, ils se 
montrent davantage allégeants que rebelles (Gangloff, Mayoral et Quiroga, 2005 b ; Mayoral, Gangloff et Duringer, 2005).

Mais l'allégeance peut se concrétiser par diverses conduites et résulter de différentes causalités. II nous a donc semblé nécessaire d'affiner les résultats antérieurement obtenus, d'une part en étudiant leurs possibilités de différenciations, et d'autre part en examinant si ces éventuelles différenciations variaient en fonction du statut du subordonné. C'est l'objet du présent travail, conduit sur une population constituée d'ouvriers et de cadres moyens argentins. Trois questions sont plus précisément au centre de la présente recherche, à savoir : 1) si ces subordonnés ont une conscience de cette norme de manière globale ou s'ils effectuent des différenciations entre ses diverses concrétisations, 2) si, spontanément, ils suivent cette norme en ses diverses concrétisations ou de manière sélective, et 3 ) si ces attitudes globalisantes ou différenciatrices des représentations et des conduites variaient en fonction du statut du subordonné concerné (cadre ou ouvrier).

\section{Procédure}

L'étude a été réalisée sur une population masculine de 180 salariés de la province de Buenos Aires répartis en deux groupes : 90 ouvriers et 90 cadres sans personnel à charge.

Les sujets de chacun de ces deux groupes furent invités à un questionnaire d'allégeance déjà employé en France (Gangloff et Caboux, 2003) et constitué de 12 items (cf. annexe). Mais nous avons, dans chacun de ces deux groupes, constitué trois sous-groupes de trente sujets : dans le $1^{\mathrm{er}}$, les sujets devaient répondre au questionnaire en tentant de donner la meilleure image possible d'eux-mêmes, dans le $2^{\text {ème }}$ ils devaient répondre de manière honnête, et dans le $3^{\text {ème }}$ ils devaient fournir des réponses censées donner la plus mauvaise image possible d'euxmêmes. La répartition des sujets dans chacun des six sous-groupes figure dans le tableau 1 suivant.

Tableau 1. Répartition des sujets en fonction de leur statut et de la consigne

\begin{tabular}{cccc}
\hline & $\begin{array}{c}\text { Consigne } \\
\text { Surnormative } \\
(\mathrm{N}=60)\end{array}$ & $\begin{array}{c}\text { Consigne } \\
\text { Honnête } \\
(\mathrm{N}=60)\end{array}$ & $\begin{array}{c}\text { Consigne } \\
\text { Contrenormative } \\
(\mathrm{N}=60)\end{array}$ \\
\hline Ouvriers $(\mathrm{N}=90)$ & $\mathrm{N}=30$ & $\mathrm{~N}=30$ & $\mathrm{~N}=30$ \\
Cadres $(\mathrm{N}=90)$ & $\mathrm{N}=30$ & $\mathrm{~N}=30$ & $\mathrm{~N}=30$ \\
\hline
\end{tabular}

Quant aux trois consignes, elles figurent plus précisément ci-dessous, telles qu'elles furent transmises respectivement aux ouvriers et aux cadres :

- Consigne sobrenormative (CS) :

"Il vous est présenté ci-dessous un questionnaire composé de 12 affirmations.

Nous vous demandons de le compléter comme s'il s'agissait d'un document que vous devez annexer à votre $\mathrm{CV}$, document qui sera présenté à un recruteur qui décidera ensuite si il vous embauche pour un poste d'ouvrier (vs de cadre). II vous est demandé d'indiquer, face à chaque affirmation, si vous êtes d'accord ou si vous n'êtes pas d'accord, en cochant les cases "d'accord" ou "pas d'accord".
Nous vous demandons enfin de répondre en tentant de donner la meilleure image possible de vous-même, comme si vous vouliez vraiment mettre toutes les chances de votre côté pour être recruté par cette entreprise".

- Consigne honnête $(\mathrm{CH})$ :

"Il vous est présenté ci-dessous un questionnaire composé de 12 affirmations.

Nous vous demandons de le compléter comme s'il s'agissait d'un document que vous devez annexer à votre $\mathrm{CV}$, document qui sera présenté à un recruteur qui décidera ensuite si il vous embauche pour un poste d'ouvrier (vs de cadre). II vous est demandé d'indiquer, face à chaque affirmation, si vous êtes d'accord ou si vous 
n'êtes pas d'accord, en cochant les cases "d'accord" ou "pas d'accord".

Nous vous demandons enfin de répondre de la manière la plus honnête possible".

- Consigne contranormative (CC):

"Il vous est présenté ci-dessous un questionnaire composé de 12 affirmations.

Nous vous demandons de le compléter comme s'il s'agissait d'un document que vous devez annexer à votre $\mathrm{CV}$, document qui sera présenté à un recruteur qui décidera ensuite si il vous embauche pour un poste d'ouvrier (vs de cadre). II vous est demandé d'indiquer, face à chaque affirmation, si vous êtes d'accord ou si vous n'êtes pas d'accord, en cochant les cases "d'accord" ou "pas d'accord".

Nous vous demandons enfin de répondre en tentant de donner la plus mauvaise image possible de vous-même, comme si vous vouliez vraiment n'avoir aucune chance d'être recruté par cette entreprise".

\section{Différences entre les items intra groupe}

Notre première analyse a consisté à calculer les taux d'approbation/désapprobation obtenus à chacun des items puis à examiner les similitudes (versus différences) de ces proportions en comparant les items deux à deux. Statistiquement parlant, nous avons utilisé le test de différences de proportions de McNemar sur échantillons appariés".

La lecture des tableaux 2 à 7 , dans chacun desquels nous avons 66 différences possibles, permet alors de remarquer 22 différences pour le groupe SOC, 26 pour le groupe HOC, 4 pour le COC, 30 pour le SMC, 29 pour le HMC et 6 pour le CMC. Cela signifie qu'il y a bien, comme nous en faisions l'hypothèse, une diversification des réponses selon les items, diversification dont l'amplitude

\footnotetext{
${ }^{4}$ L'application de ce test signifie donc calculer le nombre d'individus ayant formulé leur accord à l'une comme à l'autre de 2 questions et le nombre d'individus ayant formulé leur désaccord à l'une comme et à l'autre de ces 2 questions (mettant à part le nombre d'individus n'ayant été d'accord qu'à l'une de ces 2 questions ou n'ayant été en désaccord qu'à l'une des 2 questions). En conséquence, 2 questions peuvent aboutir à la même proportion d'accords sans pour autant que chacune se différencie d'une même troisième question (ainsi, par exemple dans le tableau 2 suivant, les questions 6 et 11 présentent des proportions identiques, mais seule la 6 aboutit à une différence significative par rapport à la 10).
}

varie, notamment en fonction de la consigne, mais aussi du statut. Nous remarquons ainsi un beaucoup plus grand nombre de différences inter-items avec les consignes surnormatives et honnêtes qu'avec les consignes contrenormatives, et également plus de différences chez les cadres que chez les ouvriers.

Par ailleurs, sachant que chaque item peut conduire à 11 différences significatives, nous avons noté les items conduisant à plus de 5 différences (c'est-à-dire à un nombre de différences supérieur à la moyenne, qui est de 5.5). Nous pouvons alors relever que se détachent : dans le groupe SOC les items 7 et 10 , dans le groupe HOC les items 10,12 et 7 , dans le groupe SMC les items $10,12,3$ et 8 , et dans le groupe HMC les items 10, 7 et 2 . C'est-à-dire que l'item se détachant le plus fréquemment des autres quels que soit la consigne et le statut du répondant est l'item 10 (qui correspond à l'acceptation du contrôle des méthodes de travail et qui conduit systématiquement, sauf avec les consignes contrenormatives, à une réponse d'acceptation).

II convient cependant d'aller plus loin dans l'analyse et de se demander tout d'abord si la variation du nombre de différences observées signifie ou non une polarisation des réponses par rapport à la moyenne théorique. Cette question fait l'objet du point 2.

\section{Différences à la moyenne théorique}

Nous nous sommes ici demandé si les réponses fournies par nos sujets différaient de la moyenne théorique, et nous avons effectué ces comparaisons grâce au test exact de Fisher. Les résultats détaillés figurent au tableau 8 et sont résumés dans le tableau 9. Nous remarquons alors notamment:

- chez les ouvriers, que 4 items donnent lieu à des réponses supérieures à la moyenne théorique aussi bien avec la consigne neutre qu'avec la surnormative : les items 1 (abandon de toute autonomie décisionnelle), 2 (exécution systématique des ordres), 7 (éviter de créer des problèmes) et 10 (acceptation du contrôle des méthodes de travail). Par ailleurs, en consigne contrenormative, l'item 2 conduit également à des réponses inférieures à la moyenne théorique. Cela signifie que cet item 2 est, dans cette population, celui qui donne lieu à la plus forte variation interconsignes, et qu'il constitue donc l'item à la plus forte claivoyance normative; 
Tableau 2. Valeurs des $p$ obtenus en cas de différences significatives (i.e. $<0,05)$ de réponses entre les 12 items du questionnaires chez les ouvriers avec emploi, avec la consigne surnormative (Surnormative Ouvriers Avec emploi). Figurent en ordonnée les moyennes des accords obtenus par question (en données brutes et en pourcentages).

\begin{tabular}{|c|c|c|c|c|c|c|c|c|c|c|c|c|}
\hline Soc & R1 & R2 & R3 & R4 & R5 & R6 & R7 & R8 & R9 & R10 & R11 & R12 \\
\hline R1 $(26 ; 87 \%)$ & & & $<0.01$ & & & & & & .022 & & & .013 \\
\hline R2 $(27 ; 90 \%)$ & & & $<0.01$ & & .022 & & & $<0.01$ & $<0.01$ & & & $<0.01$ \\
\hline R3 $(13 ; 43 \%)$ & & & & .022 & & & $<0.01$ & & & $<0.01$ & & \\
\hline R4 $(22 ; 73 \%)$ & & & & & & & & & & & & \\
\hline R5 $(18 ; 60 \%)$ & & & & & & & $<0.01$ & & & $<0.01$ & & \\
\hline R6 $(21 ; 70 \%)$ & & & & & & & .039 & & & .039 & & \\
\hline R7 $(28 ; 93 \%)$ & & & & & & & & $<0.01$ & $<0.01$ & & .039 & $<0.01$ \\
\hline R8 $(19 ; 63 \%)$ & & & & & & & & & & .012 & & \\
\hline R9 $(17 ; 57 \%)$ & & & & & & & & & & $<0.01$ & & \\
\hline R10 (28; ; 93\%) & & & & & & & & & & & & $<0.01$ \\
\hline $\mathbf{R} 11(21 ; 70 \%)$ & & & & & & & & & & & & \\
\hline R12 $(15 ; 50 \%)$ & & & & & & & & & & & & \\
\hline
\end{tabular}

Tableau 3. Valeurs des $p$ obtenus en cas de différences significatives $(i . e .<0,05)$ de réponses entre les 12 items du questionnaires chez les ouvriers avec emploi, avec la consigne honnête (Honnête Ouvriers Avec emploi). Figurent en ordonnée les moyennes des accords obtenus par question (en données brutes et en pourcentages).

\begin{tabular}{|c|c|c|c|c|c|c|c|c|c|c|c|c|}
\hline $\mathrm{HOC}$ & R1 & R2 & R3 & R4 & R5 & R6 & R7 & $\mathbf{R 8}$ & R9 & R10 & R11 & R12 \\
\hline R1 $(23 ; 77 \%)$ & & & & & .035 & & & .035 & .021 & & & $<0,01$ \\
\hline R2 $(25 ; 83 \%)$ & & & & & $<0.01$ & & & $<0.01$ & .013 & & & $<0.01$ \\
\hline R3 $(18 ; 60 \%)$ & & & & & & & .039 & & & 012 & & .013 \\
\hline R4 $(18 ; 60 \%)$ & & & & & & & .039 & & & .012 & & $<0.01$ \\
\hline R5 (14; 47\%) & & & & & & & $<0.01$ & & & $<0.01$ & & \\
\hline R6 $(20 ; 67 \%)$ & & & & & & & & & & .039 & & $<0.01$ \\
\hline R7 (26 ; 87\%) & & & & & & & & $<0.01$ & $<0.01$ & & & $<0.01$ \\
\hline R8 $(14 ; 47 \%)$ & & & & & & & & & & $<0.01$ & & \\
\hline R9 $(15 ; 50 \%)$ & & & & & & & & & & $<0.01$ & & \\
\hline R10 $(27 ; 90 \%)$ & & & & & & & & & & & $<0.01$ & $<0.01$ \\
\hline R11 $(19 ; 63 \%)$ & & & & & & & & & & & & $<0.01$ \\
\hline R12 $(8 ; 27 \%)$ & & & & & & & & & & & & \\
\hline
\end{tabular}

Tableau 4. Valeurs des $p$ obtenus en cas de différences significatives $(i . e .<0,05)$ de réponses entre les 12 items du questionnaires chez les ouvriers avec emploi, avec la consigne contrenormative (Contrenormative Ouvriers Avec emploi). Figurent en ordonnée les moyennes des accords obtenus par question (en données brutes et en pourcentages).

\begin{tabular}{|c|c|c|c|c|c|c|c|c|c|c|c|c|}
\hline $\mathrm{COC}$ & R1 & $\mathbf{R 2}$ & R3 & R4 & R5 & R6 & R7 & $\mathbf{R 8}$ & R9 & R10 & R11 & R12 \\
\hline R1 $(11 ; 37 \%)$ & & & & & & & & & & & & \\
\hline R2 $(6 ; 20 \%)$ & & & $<0.01$ & & & & & & & & & \\
\hline R3 ( $17 ; 57 \%)$ & & & & & & .012 & & .021 & $<0.01$ & & & \\
\hline R4 (14; 47\%) & & & & & & & & & & & & \\
\hline R5 (11; 37\%) & & & & & & & & & & & & \\
\hline R6 $(8 ; 27 \%)$ & & & & & & & & & & & & \\
\hline R7 (11; 37\%) & & & & & & & & & & & & \\
\hline R8 $(7 ; 23 \%)$ & & & & & & & & & & & & \\
\hline R9 $(8 ; 27 \%)$ & & & & & & & & & & & & \\
\hline R10 $(11 ; 37 \%)$ & & & & & & & & & & & & \\
\hline R11 $(10 ; 33 \%)$ & & & & & & & & & & & & \\
\hline R12 $(10 ; 33 \%)$ & & & & & & & & & & & & \\
\hline
\end{tabular}


Tableau 5. Valeurs des $p$ obtenus en cas de différences significatives $(i . e .<0,05)$ de réponses entre les 12 items du questionnaires chez les cadres avec emploi, avec la consigne surnormative (Surnormative Cadres Avec emploi). Figurent en ordonnée les moyennes des accords obtenus par question (en données brutes et en pourcentages).

\begin{tabular}{|c|c|c|c|c|c|c|c|c|c|c|c|c|}
\hline SMC & R1 & R2 & R3 & R4 & R5 & R6 & R7 & R8 & R9 & R10 & R11 & R12 \\
\hline R1 $(23 ; 77 \%)$ & & & $<0.01$ & & & & & .013 & & .031 & & $<0.01$ \\
\hline R2 $(27 ; 90 \%)$ & & & $<0.01$ & & $<0.01$ & & & $<0.01$ & $<0.01$ & & & $<0.01$ \\
\hline R3 (12; 40\%) & & & & .013 & & $<0.01$ & $<0.01$ & & & $<0.01$ & .013 & \\
\hline R4 (22 ; 73\%) & & & & & & & & & & .039 & & $<0.01$ \\
\hline R5 $(15 ; 50 \%)$ & & & & & & & $<0,01$ & & & $<0.01$ & & \\
\hline R6 $(23 ; 77 \%)$ & & & & & & & & .013 & & & & $<0.01$ \\
\hline R7 (27 ; 90\%) & & & & & & & & $<0.01$ & $<0.01$ & & & $<0.01$ \\
\hline R8 (13 ; 43\%) & & & & & & & & & & $<0.01$ & .022 & \\
\hline R9 (17 ; 57\%) & & & & & & & & & & $<0.01$ & & .012 \\
\hline R10 (29 ; 97\%) & & & & & & & & & & & .039 & $<0.01$ \\
\hline R11 $(22 ; 73 \%)$ & & & & & & & & & & & & $<0.01$ \\
\hline R12 $(8 ; 27 \%)$ & & & & & & & & & & & & \\
\hline
\end{tabular}

Tableau 6. Valeurs des $p$ obtenus en cas de différences significatives (i.e. $<0,05)$ de réponses entre les 12 items du questionnaires chez les cadres avec emploi, avec la consigne honnête (Honnête Cadres Avec emploi). Figurent en ordonnée les moyennes des accords obtenus par question (en données brutes et en pourcentages).

\begin{tabular}{lcccccccccccc}
\hline \multicolumn{1}{c}{ HMC } & R1 & R2 & R3 & R4 & R5 & R6 & R7 & R8 & R9 & R10 & R11 & R12 \\
\hline R1 $(12 ; 40 \%)$ & & $<0.01$ & & & & & .013 & & & $<0.01$ & & \\
R2 $(24 ; 80 \%)$ & & & $<0.01$ & $<0.01$ & $<0.01$ & $<0.01$ & & $<0.01$ & $<0.01$ & & $<0.01$ & $<0.01$ \\
R3 $(8 ; 27 \%)$ & & & & & & & $<0.01$ & & & $<0.01$ & & \\
R4 $(11 ; 37 \%)$ & & & & & & & $<0.01$ & & & $<0.01$ & & \\
R5 $(9 ; 30 \%)$ & & & & & & & $<0.01$ & & & $<0.01$ & & \\
R6 $(7 ; 23 \%)$ & & & & & & & $<0.01$ & & & $<0.01$ & & \\
R7 $(22 ; 73 \%)$ & & & & & & & & $<0.01$ & .013 & & .021 & $<0.01$ \\
R8 $(6 ; 20 \%)$ & & & & & & & & & & $<0.01$ & .039 & \\
R9 $(11 ; 37 \%)$ & & & & & & & & & & $<0.01$ & & \\
R10 $(25 ; 83 \%)$ & & & & & & & & & & & $<0.01$ & $<0.01$ \\
R11 $(14 ; 47 \%)$ & & & & & & & & & & & & \\
R12 $(6 ; 20 \%)$ & & & & & & & & & & & & \\
\hline
\end{tabular}

Tableau 7. Valeurs des $p$ obtenus en cas de différences significatives (i.e. $<0,05)$ de réponses entre les 12 items du questionnaires chez les cadres avec emploi, avec la consigne contrenormative (Contrenormative Cadres Avec emploi). Figurent en ordonnée les moyennes des accords obtenus par question (en données brutes et en pourcentages).

\begin{tabular}{|c|c|c|c|c|c|c|c|c|c|c|c|c|}
\hline \multirow{2}{*}{$\begin{array}{c}\text { CMC } \\
\text { R1 }(11 ; 37 \%)\end{array}$} & R1 & $\mathbf{R 2}$ & R3 & R4 & R5 & R6 & R7 & R8 & R9 & R10 & R11 & R12 \\
\hline & & & & & & & & & & & & \\
\hline R2 $(5 ; 17 \%)$ & & & $<0.01$ & & & .013 & & & & & & $<0.01$ \\
\hline R3 $(16 ; 53 \%)$ & & & & & & & & & & & .013 & \\
\hline R4 $(13 ; 43 \%)$ & & & & & & & & & & & & \\
\hline R5 $(12 ; 40 \%)$ & & & & & & & & & & & & \\
\hline R6 $(15 ; 50 \%)$ & & & & & & & & & & & .013 & \\
\hline R7 $(11 ; 37 \%)$ & & & & & & & & & & & & \\
\hline R8 $(10 ; 33 \%)$ & & & & & & & & & & & & \\
\hline R9 $(11 ; 37 \%)$ & & & & & & & & & & & & \\
\hline R10 (8 ; 27\%) & & & & & & & & & & & & \\
\hline R11 (5; 17\%) & & & & & & & & & & & & $<0.01$ \\
\hline R12 (16; 53\%) & & & & & & & & & & & & \\
\hline
\end{tabular}


- chez les cadres, que des réponses supérieures à la moyenne théorique sont obtenues pour les items 2 (éviter de créer des problèmes) et 10 (acceptation du contrôle des méthodes de travail), aussi bien en consigne surnormative qu'en consigne honnête, et que là encore l'item 2 conduit, avec la consigne contrenormative, à des réponses inférieures à la moyenne.

Tableau 8. Nombre d'accords obtenus par item (en données brutes, et entre parenthèses en pourcentages), chez les ouvriers et chez les cadres avec emploi (soit 6 groupes), avec en italiques la significativité par rapport à la moyenne théorique en cas de différence significative (chaque item donnant lieu à une cotation en 0 ou 1, la moyenne théorique par item est de 0,5 , soit, pour 30 sujets par groupe, une moyenne théorique globale par groupe de 15). La première lettre ( $\mathrm{S}, \mathrm{H}$ ou $\mathrm{C}$ ) signifie respectivement consigne Surnormative, Honnête, Contrenormative ; la $2^{\text {ème }}(\mathrm{O}$ ou $\mathrm{M})$ signifie Ouvriers ou Cadres, la $3^{\text {ème }}$ (C) signifie Avec emploi.

\begin{tabular}{|c|c|c|c|c|c|c|c|c|c|c|c|c|}
\hline & 1 & 2 & 3 & 4 & 5 & 6 & 7 & 8 & 9 & 10 & 11 & 12 \\
\hline \multirow[t]{2}{*}{ SOC } & $\begin{array}{c}26 \\
(870)\end{array}$ & 27 & $\begin{array}{c}13 \\
\end{array}$ & $\begin{array}{c}22 \\
\end{array}$ & $\begin{array}{c}18 \\
18\end{array}$ & $\begin{array}{c}21 \\
(70 \%\end{array}$ & $\begin{array}{c}28 \\
\end{array}$ & $\begin{array}{c}19 \\
193 \%\end{array}$ & $\begin{array}{c}17 \\
(57 \%)\end{array}$ & $\begin{array}{c}28 \\
\end{array}$ & $\begin{array}{c}21 \\
70 \%\end{array}$ & $\begin{array}{c}15 \\
50 \%\end{array}$ \\
\hline & $<0.01$ & $<0.01$ & & & & & $<0.01$ & & & $<0.01$ & & \\
\hline \multirow[t]{2}{*}{ SMC } & $\begin{array}{c}23 \\
(77 \%)\end{array}$ & $\begin{array}{c}27 \\
(90 \%)\end{array}$ & $\begin{array}{c}12 \\
(40 \%)\end{array}$ & $\begin{array}{c}22 \\
(73 \%)\end{array}$ & $\begin{array}{c}15 \\
(50 \%)\end{array}$ & $\begin{array}{c}23 \\
(77 \%)\end{array}$ & $\begin{array}{c}27 \\
(90 \%)\end{array}$ & $\begin{array}{c}13 \\
(43 \%)\end{array}$ & $\begin{array}{c}17 \\
(57 \%)\end{array}$ & $\begin{array}{c}29 \\
(97 \%)\end{array}$ & $\begin{array}{c}22 \\
(73 \%)\end{array}$ & $\begin{array}{c}8 \\
(27 \%)\end{array}$ \\
\hline & 0.03 & $<0.01$ & & & & 0.03 & $<0.01$ & & & $<0.01$ & & \\
\hline \multirow[t]{2}{*}{ HOC } & $\begin{array}{c}23 \\
(77 \%)\end{array}$ & $\begin{array}{c}25 \\
(83 \%)\end{array}$ & $\begin{array}{c}18 \\
(60 \%)\end{array}$ & $\begin{array}{c}18 \\
(60 \%)\end{array}$ & $\begin{array}{c}14 \\
(47 \%)\end{array}$ & $\begin{array}{c}20 \\
(67 \%)\end{array}$ & $\begin{array}{c}26 \\
(87 \%)\end{array}$ & $\begin{array}{c}14 \\
(47 \%)\end{array}$ & $\begin{array}{c}15 \\
(50 \%)\end{array}$ & $\begin{array}{c}27 \\
(90 \%)\end{array}$ & $\begin{array}{c}19 \\
(63 \%)\end{array}$ & $\begin{array}{c}8 \\
(27 \%)\end{array}$ \\
\hline & 0.03 & $<0.01$ & & & & & $<0.01$ & & & $<0.01$ & & \\
\hline \multirow[t]{2}{*}{ HMC } & $\begin{array}{c}12 \\
(40 \%)\end{array}$ & $\begin{array}{c}24 \\
(80 \%)\end{array}$ & $\mid \begin{array}{c}8 \\
27 \%)\end{array}$ & $\begin{array}{c}11 \\
(37 \%)\end{array}$ & $\begin{array}{c}9 \\
(30 \%)\end{array}$ & $\begin{array}{c}7 \\
(23 \%)\end{array}$ & $\begin{array}{c}22 \\
(73 \%)\end{array}$ & $\begin{array}{c}6 \\
(20 \%)\end{array}$ & $\begin{array}{c}11 \\
(37 \%)\end{array}$ & $\begin{array}{c}25 \\
(83 \%)\end{array}$ & $\begin{array}{c}14 \\
(47 \%)\end{array}$ & $\begin{array}{c}6 \\
(20 \%)\end{array}$ \\
\hline & & 0.01 & & & & 0.03 & & 0.01 & & $<0.01$ & & 0.01 \\
\hline \multirow[t]{2}{*}{ COC } & $\begin{array}{c}11 \\
(37 \%)\end{array}$ & $\begin{array}{c}6 \\
(20 \%)\end{array}$ & $\begin{array}{c}17 \\
(57 \%)\end{array}$ & $\begin{array}{c}14 \\
(47 \%)\end{array}$ & $\begin{array}{c}11 \\
(37 \%)\end{array}$ & $\begin{array}{c}8 \\
(27 \%)\end{array}$ & $\begin{array}{c}11 \\
(37 \%)\end{array}$ & $\begin{array}{c}7 \\
(23 \%)\end{array}$ & $\begin{array}{c}8 \\
(27 \%)\end{array}$ & $\begin{array}{c}11 \\
(37 \%)\end{array}$ & $\begin{array}{c}10 \\
(33 \%)\end{array}$ & $\begin{array}{c}10 \\
(33 \%)\end{array}$ \\
\hline & & 0.01 & & & & & & 0.03 & & & & \\
\hline CMC & $\begin{array}{c}11 \\
(37 \%)\end{array}$ & $\begin{array}{c}5 \\
(17 \%)\end{array}$ & $\begin{array}{c}16 \\
(53 \%)\end{array}$ & $\begin{array}{c}13 \\
(43 \%)\end{array}$ & $\begin{array}{c}12 \\
(40 \%)\end{array}$ & $\begin{array}{c}15 \\
(50 \%)\end{array}$ & $\begin{array}{c}11 \\
(37 \%)\end{array}$ & $\begin{array}{c}10 \\
(33 \%)\end{array}$ & $\begin{array}{c}11 \\
(37 \%)\end{array}$ & $\begin{array}{c}8 \\
(27 \%)\end{array}$ & $\begin{array}{c}5 \\
(17 \%)\end{array}$ & $\begin{array}{c}16 \\
(53 \%)\end{array}$ \\
\hline
\end{tabular}

Tableau 9. Numéro des items, pour les ouvriers et les cadres avec emploi, pour lesquels l'accord est significativement supérieur ou inférieur à la moyenne théorique

\begin{tabular}{|c|c|c|c|}
\hline & & Ouvriers & Cadres \\
\hline Surnormative & $\begin{array}{l}\text { Supérieur à la moyenne } \\
\text { Inférieur à la moyenne }\end{array}$ & $1,2,7,10$ & $1,2,6,7,10$ \\
\hline Honnête & $\begin{array}{l}\text { Supérieur à la moyenne } \\
\text { Inférieur à la moyenne }\end{array}$ & $1,2,7,10$ & $\begin{array}{c}2,10 \\
6,8,12\end{array}$ \\
\hline Contrenormative & $\begin{array}{l}\text { Supérieur à la moyenne } \\
\text { Inférieur à la moyenne }\end{array}$ & 2,8 & 2,11 \\
\hline
\end{tabular}

\section{Différences intergroupes par question}

Notre dernier questionnement a enfin porté sur les éventuelles différences de réponses inter groupes, examen là encore réalisé grâce au test exact de Fischer.

L'examen du tableau 10 nous permet alors de constater que ce sont les items 6 (l'allégeance par carriérisme), 1 (abandon de toute autonomie décisionnelle) et 11 (reconnaissance de l'expertise du chef) qui conduisent aux variations les plus fréquentes.
La variable consigne permet de constater, entre la consigne surnormative et la contrenormative, 9 différences significatives (sur 12 possibles) chez les ouvriers (SOC/COC) et 8 chez les cadres (SMC/CMC).

$\mathrm{Si}$ maintenant on se centre sur la variable statut, on remarque qu'elle ne conduit à des différences qu'avec la consigne honnête (HOC/HMC): avec les questions 1, 3 (autocensure de son propre point de vue) , 6 et 8 (reconnaissance de la légitimité du chef), les ouvriers étant, sur ces 4 items, plus allégeants que les cadres. 
Tableau 10. Valeurs des $p$ attestant d'une différence significative (i.e. quand ils sont inférieurs à 0,05 ) intergroupes, par question (ainsi, entre SOS et $\mathrm{COC}$, à la question 4, la différence est significative à $\mathrm{p}=0,04)$. La première lettre $(\mathrm{S}, \mathrm{H}$ ou $\mathrm{C})$ signifie respectivement consigne Surnormative, Honnête, Contrenormative ; la $2^{\text {ème }}$ (O ou M) signifie Ouvriers ou Cadres, la $3^{\text {ème }}$ (C) signifie Avec emploi.

\begin{tabular}{|c|c|c|c|c|c|c|c|c|c|c|c|c|c|}
\hline & & 1 & 2 & 3 & 4 & 5 & 6 & 7 & 8 & 9 & 10 & 11 & 12 \\
\hline \multicolumn{14}{|c|}{$\mathrm{SOC} \mathrm{HOC}$} \\
\hline & $\mathrm{COC}$ & $<0.01$ & $<0.01$ & & 0.04 & & $<0.01$ & $<0.01$ & $<0.01$ & 0.02 & $<0.01$ & $<0.01$ & \\
\hline & SMC & & & & & & & & & & & & \\
\hline \multirow[t]{2}{*}{$\mathrm{HOC}$} & $\mathrm{COC}$ & $<0.01$ & $<0.01$ & & & & $<0.01$ & $<0.01$ & & & $<0.01$ & 0.02 & \\
\hline & HMC & $<0.01$ & & 0.01 & & & $<0.01$ & & 0.03 & & & & \\
\hline $\mathrm{COC}$ & CMC & & & & & & & & & & & & \\
\hline \multirow[t]{2}{*}{ SMC } & HMC & $<0.01$ & & & $<0.01$ & & $<0.01$ & & & & & 0,04 & \\
\hline & CMC & $<0.01$ & $<0.01$ & & 0.02 & & 0.03 & $<0.01$ & & & $<0.01$ & $<0.01$ & 0.04 \\
\hline HMC & CMC & & $<0.01$ & 0.04 & & & 0.03 & $<0.01$ & & & $<0.01$ & 0.01 & $<0.01$ \\
\hline
\end{tabular}

\section{Discussion-Conclusion}

Les différences observées entre consignes surnormatives et consignes contrenormatives (c'est-à-dire le fait que nos sujets fournissent des réponses différences selon qu'il leur est demandé de se mettre en valeur ou de se faire mal voir) attestent qu'il y a bien clairvoyance normative. Pour autant, ces différences ne portent pas sur la totalité des items, ce qui signifie, conformément à nos hypothèses, que cette clairvoyance est sélective. Nous remarquons notamment (cf. tableau 10) que les items 3 (l'absence de défense de son propre point de vue) et 5 (l'absence de toute remarque à son supérieur hiérarchique) ne font l'objet d'aucune clairvoyance, ce quel que soit le statut du répondant (c'est-à-dire qu'il soit ouvrier ou cadre).

Nous remarquons également que, spontanément, les ouvriers et les cadres que nous avons interrogés ne répondent pas de la même manière à chacun des items, avec 26 différences inter-items dans le groupe $\mathrm{HOC}$ et 29 dans le groupe HMC (cf. tableaux 3 et 6).

Nous constatons enfin que ces résultats varient selon que l'on interroge des ouvriers ou des cadres, donnée qui confirme l'effet de cette variable statut. Nous observons en particulier, avec la consigne surnormative, que les ouvriers effectuent pratiquement $1 / 3$ de différenciations inter-items de moins que les cadres (22 contre 30 : cf. tableaux 3 et 6). Cette moindre sélectivité des ouvriers témoigne ainsi de la moins grande autonomie des ouvriers, de la moindre liberté d'appréciation des normes qu'ils s'autorisent par rapport aux cadres. Un tel résultat s'inscrit donc totalement dans les rôles respectifs attribués aux ouvriers et aux cadres, rôles notamment explicités par Taylor et dont, aujourd'hui encore, et par delà les discours négationistes, nous observons ici la vigueur phénoménologique.

\section{Bibliographie}

Caprara G.V., Barbaranelli C., et Borgogni L. (1997). Alter ego ; les 5 facteurs fondamentaux de la personnalité. Paris : E.A.P.

Gangloff B. (2000). La saturation normative des tests dits de personnalité. Communication au $27^{7 \dot{m} e}$ Congrès International de Psychologie, Stockholm (Suède).

Gangloff B. (2002). L'internalité et l'allégeance considérées comme des normes : une revue. Les Cahiers de Psychologie Politique, n2.

Gangloff B., Caboux N. (2003). Conformismo a la norma de alineación y reacciones a las injusticias profesionales. $29^{\text {ème }}$ Congreso Interamericano de Psicologia. Lima, Peru.

Gangloff B., Huet M. (2004). Poids respectif du gros cinq et de l'allégeant en situation de recrutement. Communication au 13ème Congrès International de Psychologie du Travail et de Organisations, Bologne (Italie).

Gangloff B., Mayoral L., Duringer L. (2005a). Los asalariados que prefieren los jefes argentinos : influencia del nivel de alineacion y del género, $30^{\text {ème }}$ Congrès de la Société Interaméricaine de Psychologie, Buenos Aires (Argentine).

Gangloff B., Mayoral L., Quiroga F. (2005b). La percepcion de los obreros argentinos en cuanto al nivel de alineacion conveniente para ser reclutados, $30^{\text {ème }}$ Congrès de la Société Interaméricaine de Psychologie, Buenos Aires (Argentine). 
Mayoral L., Gangloff B., Duringer L. (2005). La percepcion de los mandos medios argentinos en cuanto al nivel de alineacion conveniente para ser reclutados, $30^{\text {ème }}$ Congrès de la Société Interaméricaine de Psychologie, Buenos Aires (Argentine).

Py J., Somat A. (1991). Normativité, conformité et clairvoyance : leurs effets sur le jugement évaluatif dans un contexte scolaire. In : J-L. Beauvois, R-V. Joule et J-M. Monteil (Eds),
Perpectives cognitives et conduites sociales. Cousset : Del Val, vol. 3, 167-193.

Rolland J-P. (1994). Désirabilité sociale de marqueurs des dimensions de ma personnalité du modèle en 5 facteurs. Revue Européenne de Psychologie Apliquée, 44(1), 65-71

\section{Annexe}

\section{Le questionnaire utilisé}

Ce questionnaire comprend 9 items portant sur les manifestations de l'allégeance (items 1, 2, 3, 4, 5, 7, 9, 10,12 ) et 3 items portant sur les raisons de l'allégeance (items 6, 8, 11). Est indiquée ci-dessous entre parenthèses et en gras la thématique de chacun des items (ces thématiques ne figuraient évidemment pas dans les questionnaires remis aux sujets) :

1. Au travail, il me semble normal de demander systématiquement l'accord de mon chef avant de prendre une décision (abandon de toute autonomie décisionnelle).

2. Dans mon travail, il me paraît normal d'exécuter tous les ordres que me donne mon supérieur (exécution systématique des ordres).

3. Au travail, avec mon chef, je tente rarement de défendre mes idées (non défense de son propre point de vue)

4. Au travail, quand la direction annonce ses choix, il est rare que je me plaigne ouvertement (non questionnement des décisions).

5. Au travail, j'évite de faire des remarques à mon supérieur, même si, dans certaines situations, il le mérite (absence de toute remarque).

6. Au travail, je trouve normal d'accepter toutes les décisions de mes supérieurs si je veux gravir les échelons (carriérisme).

7. Au travail, quelles que soient les conditions de travail, je trouve normal d'éviter de créer des problèmes (évitement de la création de problèmes).

8. Au travail, j'accepte plus facilement un ton autoritaire quand il vient de mon chef (reconnaissance de la légitimité du chef).

9. Au travail, dans toutes circonstances, je trouve normal que l'avis de mon supérieur soit plus important que le mien (acceptation de la prédominance du point de vue du chef).

10. Au travail, je trouve normal que mon supérieur puisse à n'importe quel moment vérifier si je respecte les méthodes de travail (acceptation du contrôle des méthodes de travail).

11. Au travail, je me dis souvent que mon patron est le mieux placé pour savoir ce qui est bon pour son entreprise (reconnaissance de l'expertise du chef).

12. Au travail, je trouve normal que mon patron ne justifie pas ses décisions auprès de moi, même si elles me concernent directement (renoncement à demander justification des décisions). 